\title{
Correlated Relation between Quantum Discord and Entanglement of Two-Atom in Thermal Reservoirs
}

\author{
Xue-Qun Yan and Zhuan-Lin Yue \\ Department of Physics, School of Science, Tianjin Polytechnic University, Tianjin 300387, China \\ Correspondence should be addressed to Xue-Qun Yan; xqyan867@tom.com
}

Received 6 December 2013; Revised 18 March 2014; Accepted 19 March 2014; Published 10 April 2014

Academic Editor: Keli Han

Copyright (c) 2014 X.-Q. Yan and Z.-L. Yue. This is an open access article distributed under the Creative Commons Attribution License, which permits unrestricted use, distribution, and reproduction in any medium, provided the original work is properly cited.

\begin{abstract}
We study the time evolution of quantum discord and entanglement of a two-qubit system in thermal reservoirs. We find that there are no simple ordering relations between entanglement and quantum discord for the dynamical evolution behavior; that is, quantum discord may be smaller or larger than entanglement in the evolution process. However, a strong correlation between changes of the ordering relations and the mean photon number is found. In addition, it also shows that entanglement is not the strongest form of nonclassicality.
\end{abstract}

Considerable efforts have been devoted to study quantum correlations in the last two decades, mainly due to the general belief that they are a fundamental resource for quantum information processing tasks. Among them, entanglement is the most famous and best studied kind of quantum correlation and is generally regarded as a necessary resource in quantum computation and communication [1]. The situation started to change after a so-called DQC1 (deterministic quantum computation with one qubit) model was presented which may provide the speedup in the deterministic quantum computation with one pure qubit [2]. The fact is that no entanglement is present in this model; however, other kinds of nonclassical correlations are responsible for the quantum computational efficiency of DQC1. Such correlations are characterized as quantum discord [3], which is believed to be more general and more fundamental than entanglement [4-6] and which can be viewed as the amount of entanglement consumed in the quantum-state merging $[7,8]$.

Besides its application in DQC1, quantum discord has also been used in studies of quantum phase transition $[9,10]$, Maxwell's demon [11], and relativistic effect [12, 13]. In addition, concerning biological systems, it has been suggested that such correlations could play an important role in photosynthesis [14]. In particular, quantum discord has been predicted to show peculiar dynamics under decoherence [15]. Considering two noninteracting qubits, it was shown that, under certain conditions, the decay rate of the correlations may suffer a sudden change [16, 17]. Furthermore, by analyzing various dissipative channels, some authors have shown that, in all cases where entanglement suddenly disappears, quantum discord vanishes only asymptotically [18-20]. In this sense, quantum discord is more robust against decoherence than entanglement so that quantum algorithms based only on quantum discord correlations may be more robust than those based on entanglement. Despite intense research over the last decade, the relation between entanglement and quantum discord is not clear, and there are still a number of challenging outstanding problems that drive much effort in the field.

In this paper, we consider a two-qubit system interacting with two independent thermal reservoirs. We will explore the relation between the dynamics of entanglement and that of quantum discord of two atoms in their local reservoirs. The common belief is that measures of quantum discord should be more than those of entanglement. However, our results will show that quantum discord may be smaller or larger than entanglement in the evolution process, and there exists an interesting variation in the ordering relations between entanglement and quantum discord. Moreover, it is 
also shown that entanglement is not the strongest form of nonclassicality, as pointed out in [21].

For a two-qubit quantum system, the quantum discord is defined as the difference between the quantum mutual information and the classical correlation,

$$
D\left(\rho_{a b}\right)=I\left(\rho_{a b}\right)-C^{\prime}\left(\rho_{a b}\right)
$$

Here, the quantum mutual information of two subsystems is given by

$$
I\left(\rho_{a b}\right)=S\left(\rho_{a}\right)+S\left(\rho_{b}\right)-S\left(\rho_{a b}\right),
$$

and $S\left(\rho_{j}\right)$ is the entropy of the quantum system, which is given by the von Neumann entropy,

$$
S\left(\rho_{j}\right)=-\operatorname{Tr}_{j}\left(\rho_{j} \log _{2} \rho_{j}\right)=-\sum_{i} \lambda_{j}^{i} \log _{2} \lambda_{j}^{i}
$$

where the subscript $j$ indicates either the subsystem $a(b)$ or the total system and $\left\{\lambda_{j}^{i}\right\}$ are the nonzero eigenvalues of the quantum state $\rho_{j}$. $C^{\prime}\left(\rho_{a b}\right)$ is the classical correlation between the two subsystems [4]. As discussed in [15], the classical correlation is given by

$$
C^{\prime}\left(\rho_{a b}\right)=S\left(\rho_{a}\right)-\min _{\left\{\Pi_{k}\right\}}\left[S\left(\rho_{a b} \mid\left\{\Pi_{k}\right\}\right)\right]
$$

where the minimum is taken over the set of projective measurements $\left\{\prod_{k}\right\}$ and $S\left(\rho_{a b} \mid\left\{\prod_{k}\right\}\right)=\sum_{k} p_{k} S\left(\rho_{k}\right)$ is the quantum conditional entropy of $a$ given the complete measurements on subsystem $b$, with $\rho_{k}=\operatorname{Tr}_{b}\left(\prod_{k} \rho_{a b} \prod_{k}\right) / p_{k}$ and $p_{k}=\operatorname{Tr}_{a b}\left(\rho_{a b} \prod_{k}\right)$.

Then, quantum discord is given by

$$
D\left(\rho_{a b}\right)=S\left(\rho_{b}\right)-S\left(\rho_{a b}\right)+\min _{\left\{\Pi_{k}\right\}} S\left(\rho_{a b} \mid\left\{\Pi_{k}\right\}\right) .
$$

The evaluation of quantum discord is a hard task due to the minimization over all possible measurements. Although this cannot be done in full generality analytically even for two qubits, the problem is tractable using analytical tools for certain subsets of states (such as $X$ states) [22, 23].

The density matrix of a two-qubit $X$ state written in the standard basis is given by

$$
\rho(t)=\left(\begin{array}{cccc}
\rho_{11} & 0 & 0 & \rho_{14} \\
0 & \rho_{22} & \rho_{23} & \\
0 & \rho_{32} & \rho_{33} & 0 \\
\rho_{41} & 0 & 0 & \rho_{44}
\end{array}\right) .
$$

The eigenvalues of the matrix $\rho(t)$ in (6) can be obtained as follows:

$$
\begin{aligned}
& \lambda_{0,1}=\frac{1}{2}\left[\left(\rho_{11}+\rho_{44}\right) \pm \sqrt{\left(\rho_{11}-\rho_{44}\right)^{2}+4\left|\rho_{14}\right|^{2}}\right], \\
& \lambda_{2,3}=\frac{1}{2}\left[\left(\rho_{22}+\rho_{33}\right) \pm \sqrt{\left(\rho_{22}-\rho_{33}\right)^{2}+4\left|\rho_{23}\right|^{2}}\right]
\end{aligned}
$$

and the von Neumann entropies of the reduced density matrix of the $\rho(t)$ can be easily obtained as

$$
\begin{aligned}
S\left(\rho_{a}\right)=-[ & \left(\rho_{11}+\rho_{22}\right) \log _{2}\left(\rho_{11}+\rho_{22}\right) \\
& \left.+\left(\rho_{33}+\rho_{44}\right) \log _{2}\left(\rho_{33}+\rho_{44}\right)\right], \\
S\left(\rho_{b}\right)=-[ & \left(\rho_{11}+\rho_{33}\right) \log _{2}\left(\rho_{11}+\rho_{33}\right) \\
& \left.+\left(\rho_{22}+\rho_{44}\right) \log _{2}\left(\rho_{22}+\rho_{44}\right)\right] .
\end{aligned}
$$

The quantum conditional entropy in (4) is given by

$$
S\left(\rho_{a b} \mid\left\{\Pi_{k}\right\}\right)=p_{0} S\left(\rho_{0}\right)+p_{1} S\left(\rho_{1}\right)
$$

where

$$
\begin{aligned}
& p_{0}=\left[\left(\rho_{11}+\rho_{33}\right) k+\left(\rho_{22}+\rho_{44}\right) l\right], \\
& p_{1}=\left[\left(\rho_{11}+\rho_{33}\right) l+\left(\rho_{22}+\rho_{44}\right) k\right],
\end{aligned}
$$

and the entropies are given by

$$
\begin{gathered}
S\left(\rho_{0}\right)=-\frac{1-\theta}{2} \log _{2} \frac{1-\theta}{2}-\frac{1+\theta}{2} \log _{2} \frac{1+\theta}{2}, \\
S\left(\rho_{1}\right)=-\frac{1-\theta^{\prime}}{2} \log _{2} \frac{1-\theta^{\prime}}{2}-\frac{1+\theta^{\prime}}{2} \log _{2} \frac{1+\theta^{\prime}}{2},
\end{gathered}
$$

with

$$
\begin{aligned}
& \theta=\sqrt{\frac{\left[\left(\rho_{11}-\rho_{33}\right) k+\left(\rho_{22}-\rho_{44}\right) l\right]^{2}+\Theta}{\left[\left(\rho_{11}+\rho_{33}\right) k+\left(\rho_{22}+\rho_{44}\right) l\right]^{2}}}, \\
& \theta^{\prime}=\sqrt{\frac{\left[\left(\rho_{11}-\rho_{33}\right) l+\left(\rho_{22}-\rho_{44}\right) k\right]^{2}+\Theta}{\left[\left(\rho_{11}+\rho_{33}\right) l+\left(\rho_{22}+\rho_{44}\right) k\right]^{2}}},
\end{aligned}
$$

where $\Theta=4 k l\left[\left|\rho_{14}\right|^{2}+\left|\rho_{23}\right|^{2}+2 \operatorname{Re}\left(\rho_{14} \rho_{23}\right)\right]-16 m \operatorname{Re}\left(\rho_{14} \rho_{23}\right)+$ $16 n \operatorname{Im}\left(\rho_{14} \rho_{23}\right)$ and $\operatorname{Re}(z)$ and $\operatorname{Im}(z)$ are the real and imaginary parts of the complex number $z$.

The minimum value of $S\left(\rho_{a b} \mid\left\{\Pi_{k}\right\}\right)$ in (4) depends on parameters $k, l, m$, and $n$, where $k \in[0,1], m \in[0,1 / 4]$, $n \in[-1 / 8,1 / 8]$, and $k+l=1[22]$.

To investigate entanglement dynamics, we use the concurrence as the measure [24]. For a pair of qubits, the concurrence is defined as

$$
C(\rho)=\max \left\{0, \sqrt{\lambda_{1}^{\prime}}-\sqrt{\lambda_{2}^{\prime}}-\sqrt{\lambda_{3}^{\prime}}-\sqrt{\lambda_{4}^{\prime}}\right\},
$$

where the $\sqrt{\lambda_{i}^{\prime}}$ are the eigenvalues ( $\lambda_{1}^{\prime}$ being the largest one) of non-Hermitian matrix $\rho \tilde{\rho}$ and $\tilde{\rho}$ is defined as

$$
\tilde{\rho}=\left(\sigma_{y} \otimes \sigma_{y}\right) \rho^{*}\left(\sigma_{y} \otimes \sigma_{y}\right)
$$

with $\rho^{*}$ being the complex conjugate of matrix $\rho$. Here, $\sigma_{y}$ stands for the off-diagonal pure imaginary Pauli matrix. The concurrence $C=0$ corresponds to an unentangled state and $C=1$ corresponds to a maximally entangled state. 
In the case of the density matrix with the $\mathrm{X}$ type (6), one easily finds [25] that the concurrence is given by

$$
C=\max \left\{0, \Lambda_{1}, \Lambda_{2}\right\},
$$

where $\Lambda_{1}=2\left(\sqrt{\rho_{23} \rho_{32}}-\sqrt{\rho_{11} \rho_{44}}\right), \Lambda_{2}=2\left(\sqrt{\rho_{14} \rho_{41}}-\right.$ $\left.\sqrt{\rho_{22} \rho_{33}}\right)$.

Next, we investigate the dynamics of quantum discord and entanglement of the two atoms which are each trapped separately in one of the two spatially separated thermal reservoirs. According to the general quantum reservoir theory [26], we can derive the following equation of motion for the reduced density matrix of the atoms interacting with their local thermal reservoirs of mean thermal photon numbers $m$ and $n$ :

$$
\begin{aligned}
\frac{d \rho}{d t}= & -\frac{1}{2} \gamma_{1}(m+1)\left[\sigma_{1}^{+} \sigma_{1} \rho-2 \sigma_{1} \rho \sigma_{1}^{+}+\rho \sigma_{1}^{+} \sigma_{1}\right] \\
& -\frac{1}{2} \gamma_{1} m\left[\sigma_{1} \sigma_{1}^{+} \rho-2 \sigma_{1}^{+} \rho \sigma_{1}+\rho \sigma_{1} \sigma_{1}^{+}\right] \\
& -\frac{1}{2} \gamma_{2}(n+1)\left[\sigma_{2}^{+} \sigma_{2} \rho-2 \sigma_{2} \rho \sigma_{2}^{+}+\rho \sigma_{2}^{+} \sigma_{2}\right] \\
& -\frac{1}{2} \gamma_{2} n\left[\sigma_{2} \sigma_{2}^{+} \rho-2 \sigma_{2}^{+} \rho \sigma_{2}+\rho \sigma_{2} \sigma_{2}^{+}\right],
\end{aligned}
$$

where $\gamma_{i}$ is the spontaneous emission rate of atom $i$ and $\sigma_{i}^{+}, \sigma_{i}$ are the usual Pauli raising and lowering operators of atom $i$. Here, we assume that the correlation time between the atoms and the reservoirs is much shorter than the characteristic time of the dynamic evolution of the atoms so that the Markov approximation is valid.

In what follows, we will focus on the initial Bell states since such states provide important test ground for the understanding of nonclassicality beyond entanglement and its interplay with entanglement itself.

Now, we would like to consider Bell state $\left|\Phi^{+}\right\rangle=$ $(1 / \sqrt{2})(|10\rangle+|01\rangle)$ as the initial state. For simplicity, we assume $m=n$ and $\gamma_{1}=\gamma_{2}=\gamma$. The solution of master equation (16) is given by

$$
\begin{gathered}
\rho_{11}=\frac{1}{2(2 n+1)^{2}}\left\{2 n^{2}+2 n e^{-(2 n+1) \gamma t}\right. \\
\left.-2 n(n+1) e^{-2(2 n+1) \gamma t}\right\}, \\
\rho_{22}=\rho_{33}=\frac{1}{2(2 n+1)^{2}}\left\{2 n(n+1)+e^{-(2 n+1) \gamma t}\right. \\
\left.+2 n(n+1) e^{-2(2 n+1) \gamma t}\right\}, \\
\rho_{44}=\frac{1}{2(2 n+1)^{2}}\left\{2(n+1)^{2}-2(n+1) e^{-(2 n+1) \gamma t}\right. \\
\left.-2 n(n+1) e^{-2(2 n+1) \gamma t}\right\}, \\
\rho_{23}=\rho_{32}=\frac{1}{2} e^{-(2 n+1) \gamma t} .
\end{gathered}
$$

Using (17), we can give the eigenvalues of the matrix discussed:

$$
\begin{aligned}
& \lambda_{0,1}=\frac{1}{2}\left[\left(\rho_{11}+\rho_{44}\right) \pm\left|\rho_{11}-\rho_{44}\right|\right], \\
& \lambda_{2,3}=\rho_{22} \pm \rho_{23} .
\end{aligned}
$$

Based on the discussion of the above, we can now calculate the classical correlation and quantum discord. The quantum discord is given by

$$
\begin{aligned}
D\left(\rho_{a b}\right)= & S\left(\rho_{b}\right)-S\left(\rho_{a b}\right) \\
& +\min _{\left\{\Pi_{k}\right\}}\left\{\left.S\left(\rho_{0}\right)\right|_{\theta_{1}},\left.S\left(\rho_{a b} \mid\left\{\Pi_{k}\right\}\right)\right|_{\theta_{2}, \theta_{3}}\right\},
\end{aligned}
$$

where

$$
\begin{gathered}
\left.S\left(\rho_{0}\right)\right|_{\theta_{1}}=-\frac{1+\theta_{1}}{2} \log \frac{1+\theta_{1}}{2}-\frac{1-\theta_{1}}{2} \log _{2} \frac{1-\theta_{1}}{2}, \\
\left.S\left(\rho_{a b} \mid\left\{\Pi_{k}\right\}\right)\right|_{\theta_{2}, \theta_{3}} \\
=\left(\rho_{22}+\rho_{44}\right)\left(-\frac{1+\theta_{2}}{2} \log _{2} \frac{1+\theta_{2}}{2}-\frac{1-\theta_{2}}{2} \log _{2} \frac{1-\theta_{2}}{2}\right) \\
+\left(\rho_{11}+\rho_{33}\right)\left(-\frac{1+\theta_{3}}{2} \log _{2} \frac{1+\theta_{3}}{2}-\frac{1-\theta_{3}}{2} \log _{2} \frac{1-\theta_{3}}{2}\right),
\end{gathered}
$$

with $\theta_{1}=\sqrt{\left(\rho_{11}-\rho_{44}\right)^{2}+4\left|\rho_{23}\right|^{2}}, \theta_{2}=\left(\left|\rho_{22}-\rho_{44}\right| /\left(\rho_{22}+\rho_{44}\right)\right)$, and $\theta_{3}=\left(\left|\rho_{11}-\rho_{33}\right| /\left(\rho_{11}+\rho_{33}\right)\right)$.

Using (15) and (19), the expressions of the entanglement and quantum discord can be numerically calculated. In Figure 1, the dynamics of entanglement and quantum discord are plotted as a function of the scaled time $\gamma t$ and mean photon number $n$. Firstly, we note that quantum discord only disappears in the asymptotic limit, while the entanglement suffers a sudden death at a certain parameterized time $t_{d}$ in a thermal reservoir of nonzero mean photon number. Considering the fact that the entanglement sudden death (ESD) was firstly reported in [27], thereafter, many works have been devoted to the related topics [28-31]. We also see that both time of ESD and vanishing of quantum discord decrease as the mean thermal photon number becomes large, as expected.

In order to explore the relation between entanglement and quantum discord, we plot the evolution of both correlations for different values of the mean photon numbers $n=1$, $0.1,0.01,0$ in Figure 2. The abscissa of the vertical dotted line corresponds to the scaled time $\gamma t^{\prime}$ of the intersection point of the two evolution curves. From Figures 2(a)-2(c), one can see a similar phenomenon that the two correlations have a different order as functions of time $\gamma t$, with concurrence being initially larger than quantum discord for $0 \leq \gamma t \leq$ $\gamma t^{\prime}$, but, for $\gamma t \geq \gamma t^{\prime}$, quantum discord becomes larger than concurrence. It means that entanglement is not the strongest form of nonclassicality. Although there are no simple ordering relations between the two correlations, it is instructive to note that the position of the scaled time 


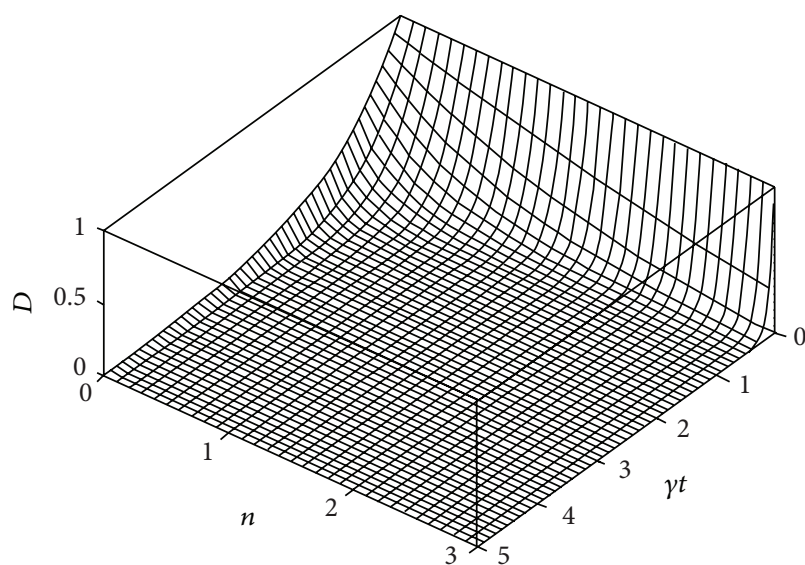

(a)

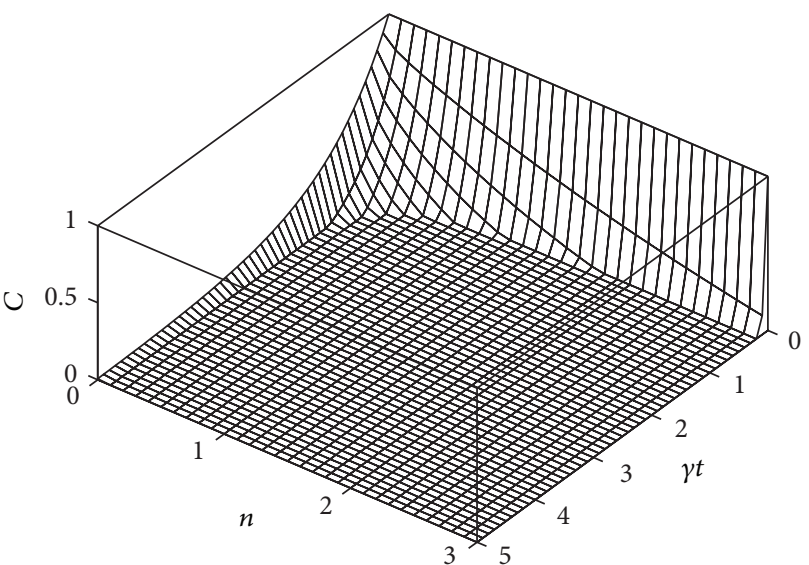

(b)

FIgURE 1: Dynamics of the quantum discord (a) and entanglement (b) as a function of the scaled time $\gamma t$ and mean photon number $n$.

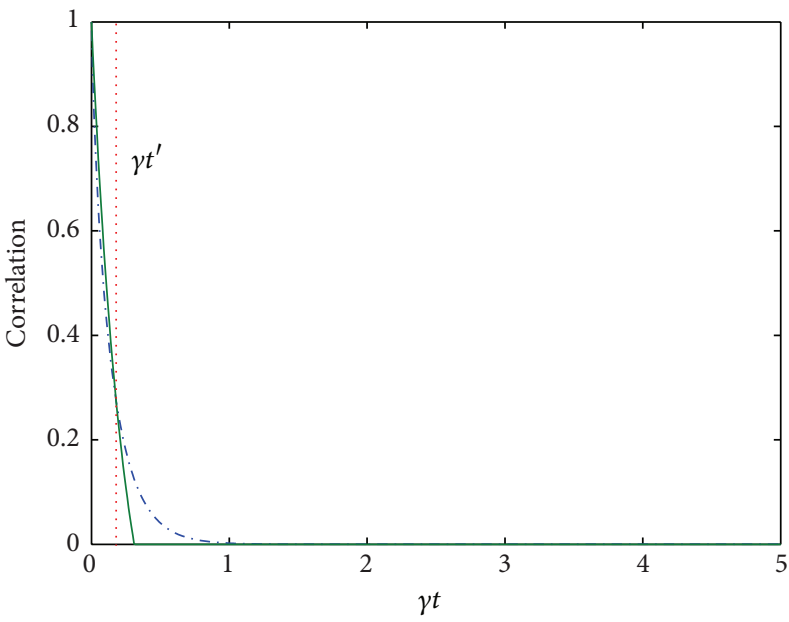

(a)

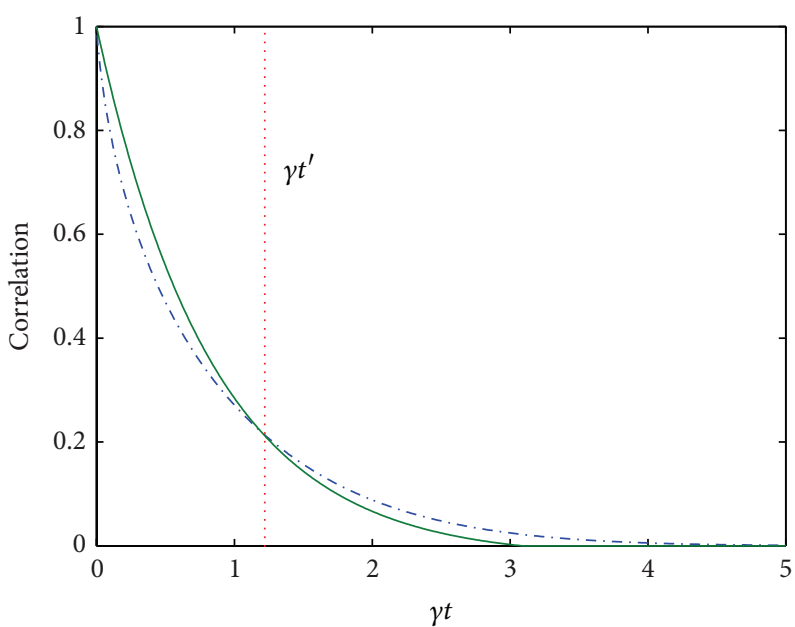

(c)

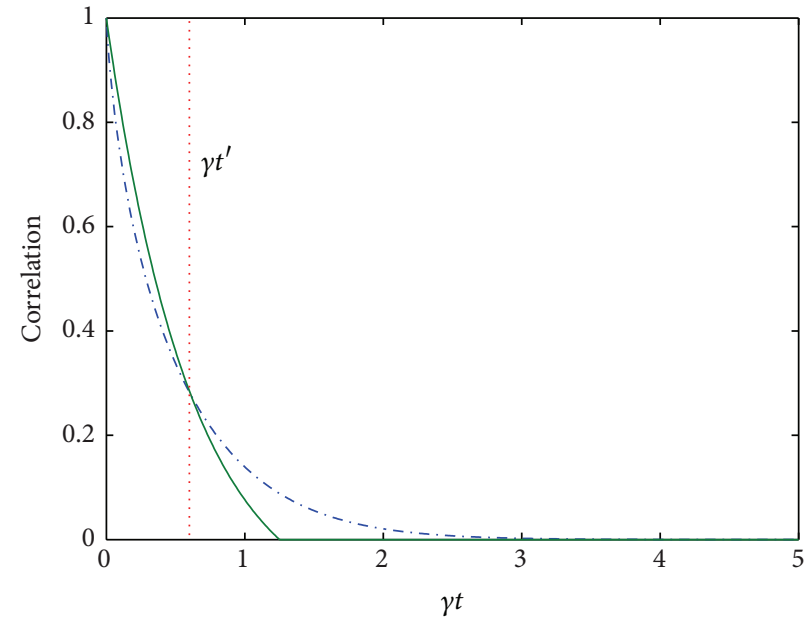

(b)

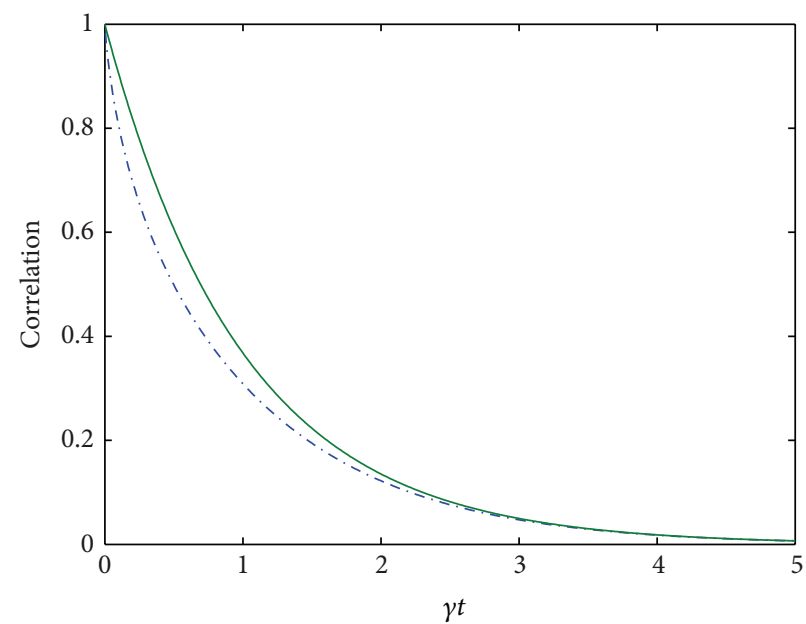

(d)

FIGURE 2: Time dependence of quantum discord (dot-dashed line) and concurrence (solid line) for mean photon number: (a) $n=1$, (b) $n=0.1$, (c) $n=0.01$, and (d) $n=0$ (color online). The abscissa of the vertical dotted line corresponds to the scaled time $\gamma t^{\prime}$ of the intersection point of the two evolution curves. 
$\gamma t^{\prime}$ of the intersection point of the two evolution curves moves to the right along the $\gamma t$-axis with the decreases of the mean photon number $n$. Moreover, one can observe from Figure 2(d) that, for $n=0, \gamma t^{\prime} \rightarrow \infty$. It can also be found that the dynamical behavior of the entanglement tends to agree with that of the quantum discord in time in the vacuum reservoirs. Meanwhile, it also implies that the ESD vanishes as the mean thermal photon number is zero. These results show that further and deeper study of the dynamical behaviors of quantum discord and entanglement in thermal environments might help to better understand the nature of quantum correlations.

In summary, we have investigated the dynamics of the entanglement and the quantum discord for two atoms coupled to independent thermal reservoirs. We found that there are no simple ordering relations between entanglement and quantum discord for the dynamical evolution behavior; however, the position of the intersection point of the two correlation curves moves to the right along the $\gamma t$-axis as the mean photon number becomes small. We also observed that, in thermal environments where entanglement suddenly disappears, quantum discord vanishes only in the asymptotic limit and the dynamical behavior of quantum discord tends to agree with that of entanglement in time in the vacuum reservoir. Our results also show that entanglement is not the strongest form of nonclassicality. It suggests that quantum algorithms based on quantum discord, different from those based on entanglement, are more resistant to external perturbations and, therefore, introduce new hope of implementing an efficient quantum algorithm and quantum information processing.

\section{Conflict of Interests}

The authors declare that there is no conflict of interests regarding the publication of this paper.

\section{Acknowledgment}

The authors are grateful to Dr. Z. Qi for careful reading of the original paper.

\section{References}

[1] M. A. Nielsen and I. L. Chuang, Quantum Computation and Quantum Information, Cambridge University Press, Cambridge, UK, 2000.

[2] E. Knill and R. Laflamme, "Power of one bit of quantum information," Physical Review Letters, vol. 81, no. 25, pp. 5672$5675,1998$.

[3] H. Ollivier and W. H. Zurek, "Quantum discord: a measure of the quantumness of correlations," Physical Review Letters, vol. 88, no. 1, Article ID 017901, 2002.

[4] L. C. Céleri, J. Maziero, and R. M. Serra, "Theoretical and experimental aspects of quantum discord and related measures," International Journal of Quantum Information, vol. 9, no. 7-8, pp. 1837-1873, 2011.

[5] R. Tatham, L. Mišta, G. Adesso, and N. Korolkova, "Nonclassical correlations in continuous-variable non-Gaussian Werner states," Physical Review A-Atomic, Molecular, and Optical Physics, vol. 85, no. 2, Article ID 022326, 2012.

[6] K. Modi, A. Brodutch, H. Cable, T. Paterek, and V. Vedral, "The classical-quantum boundary for correlations: discord and related measures," Reviews of Modern Physics, vol. 84, no. 4, pp. 1655-1707, 2012.

[7] D. Cavalcanti, L. Aolita, S. Boixo, K. Modi, M. Piani, and A. Winter, "Operational interpretations of quantum discord," Physical Review A-Atomic, Molecular, and Optical Physics, vol. 83, no. 3, Article ID 032324, 2011.

[8] V. Madhok and A. Datta, "Interpreting quantum discord through quantum state merging," Physical Review A-Atomic, Molecular, and Optical Physics, vol. 83, no. 3, Article ID 032323, 2011.

[9] M. S. Sarandy, "Classical correlation and quantum discord in critical systems," Physical Review A-Atomic, Molecular, and Optical Physics, vol. 80, no. 2, Article ID 022108, 2009.

[10] T. Werlang, C. Trippe, G. A. P. Ribeiro, and G. Rigolin, "Quantum correlations in spin chains at finite temperatures and quantum phase transitions," Physical Review Letters, vol. 105, no. 9, Article ID 095702, 2010.

[11] W. H. Zurek, "Quantum discord and Maxwell's demons," Physical Review A-Atomic, Molecular, and Optical Physics, vol. 67, no. 1, Article ID 012320, 2003.

[12] A. Datta, "Quantum discord between relatively accelerated observers," Physical Review A-Atomic, Molecular, and Optical Physics, vol. 80, no. 5, Article ID 052304, 2009.

[13] J. C. Wang, J. F. Deng, and J. L. Jing, "Classical correlation and quantum discord sharing of Dirac fields in noninertial frames," Physical Review A-Atomic, Molecular, and Optical Physics, vol. 81, no. 5, Article ID 052120, 2010.

[14] K. Brádler, M. M. Wilde, S. Vinjanampathy, and D. B. Uskov, "Identifying the quantum correlations in light-harvesting complexes," Physical Review A-Atomic, Molecular, and Optical Physics, vol. 82, no. 6, Article ID 062310, 2010.

[15] J. Maziero, L. C. Céleri, R. M. Serra, and V. Vedral, "Classical and quantum correlations under decoherence," Physical Review A-Atomic, Molecular, and Optical Physics, vol. 80, no. 4, Article ID 044102, 2009.

[16] L. Mazzola, J. Piilo, and S. Maniscalco, "Sudden transition between classical and quantum decoherence," Physical Review Letters, vol. 104, no. 20, Article ID 200401, 2010.

[17] Q. L. He, J. B. Xu, D. X. Yao, and Y. Q. Zhang, "Sudden transition between classical and quantum decoherence in dissipative cavity QED and stationary quantum discord," Physical Review A-Atomic, Molecular, and Optical Physics, vol. 84, no. 2, Article ID 022312, 2011.

[18] J. Maziero, T. Werlang, F. F. Fanchini, L. C. Céleri, and R. M. Serra, "System-reservoir dynamics of quantum and classical correlations," Physical Review A-Atomic, Molecular, and Optical Physics, vol. 81, no. 2, Article ID 022116, 2010.

[19] X. Q. Yan and Z. L. Yue, "Dynamics of quantum and classical correlations of a two-atom system in thermal reservoirs," Chaos, Solitons \& Fractals, vol. 57, pp. 117-122, 2013.

[20] R. Tanaś, "Evolution of quantum correlations in a two-atom system," Physica Scripta, vol. T153, Article ID 014059, 2013.

[21] M. Piani, S. Gharibian, G. Adesso, J. Calsamiglia, P. Horodecki, and A. Winter, "All nonclassical correlations can be activated into distillable entanglement," Physical Review Letters, vol. 106, no. 22, pp. 220403-220407, 2011. 
[22] M. Ali, A. R. P. Rau, and G. Alber, "Quantum discord for two-qubit X states," Physical Review A-Atomic, Molecular, and Optical Physics, vol. 81, no. 4, Article ID 042105, 2010.

[23] S. Luo, "Quantum discord for two-qubit systems," Physical Review A-Atomic, Molecular, and Optical Physics, vol. 77, no. 4, Article ID 042303, 2008.

[24] W. K. Wootters, "Entanglement of formation of an arbitrary state of two qubits," Physical Review Letters, vol. 80, no. 10, pp. 2245-2248, 1998.

[25] M. Ikram, F. Li, and M. S. Zubairy, "Disentanglement in a twoqubit system subjected to dissipation environments," Physical Review A-Atomic, Molecular, and Optical Physics, vol. 75, no. 6, Article ID 062336, 2007.

[26] M. O. Scully and M. S. Zubairy, Quantum Optics, Cambridge University, England, Cambridge, 1997.

[27] T. Yu and J. H. Eberly, "Finite-time disentanglement via spontaneous emission," Physical Review Letters, vol. 93, no. 14, pp. 1-140404, 2004.

[28] K. Roszak, P. Horodecki, and R. Horodecki, "Sudden death of effective entanglement," Physical Review A-Atomic, Molecular, and Optical Physics, vol. 81, no. 4, Article ID 042308, 2010.

[29] X. Q. Yan, "Entanglement sudden death of two atoms successive passing a cavity," Chaos, Solitons and Fractals, vol. 41, no. 4, pp. 1645-1650, 2009.

[30] B. Bellomo, R. Lo Franco, and G. Compagno, "Non-Markovian effects on the dynamics of entanglement," Physical Review Letters, vol. 99, no. 16, Article ID 160502, 2007.

[31] Z. Ficek and R. Tanaś, "Dark periods and revivals of entanglement in a two-qubit system," Physical Review A-Atomic, Molecular, and Optical Physics, vol. 74, no. 2, Article ID 024304, 2006. 

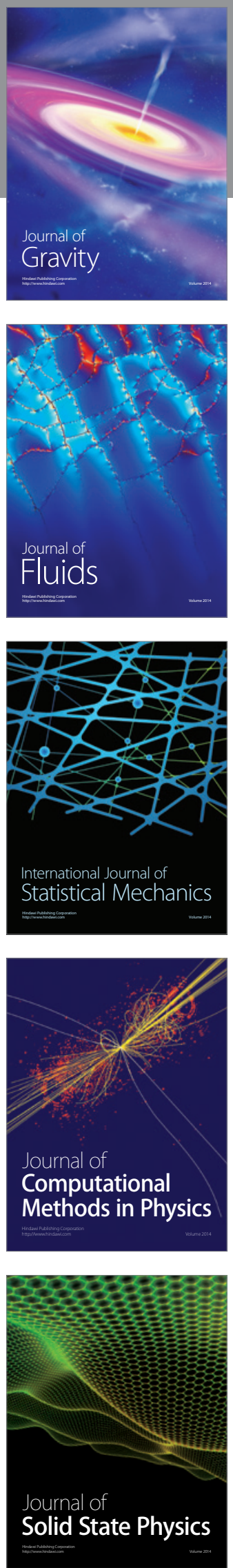

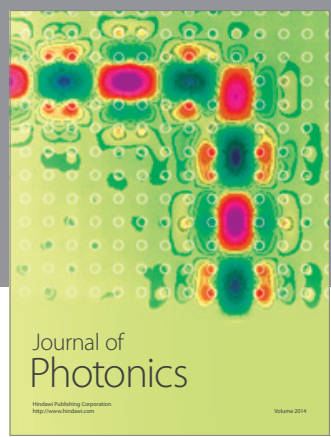

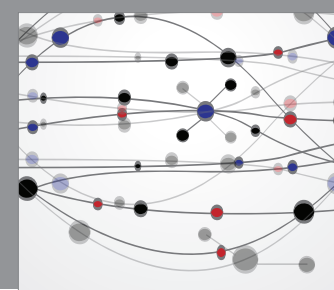

The Scientific World Journal

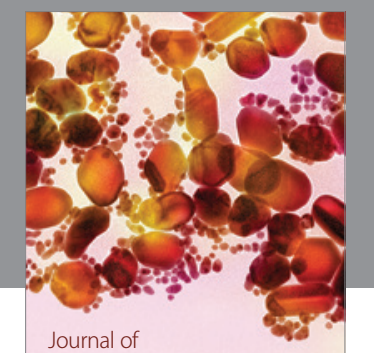

Soft Matter
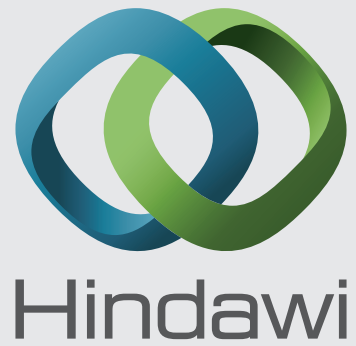

Submit your manuscripts at

http://www.hindawi.com
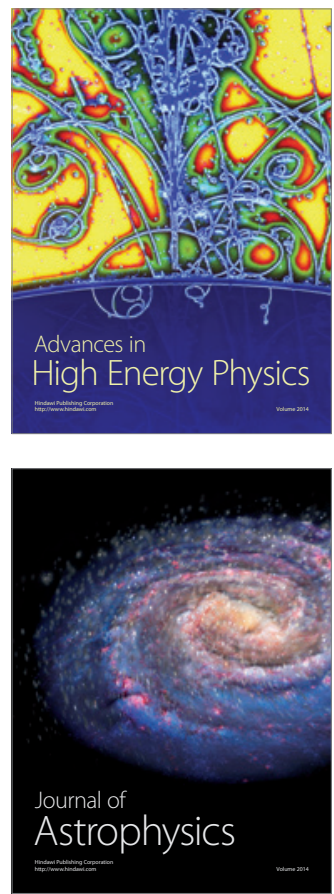
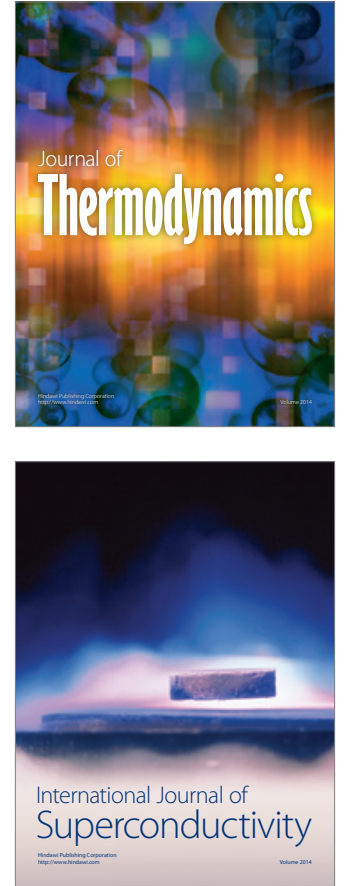
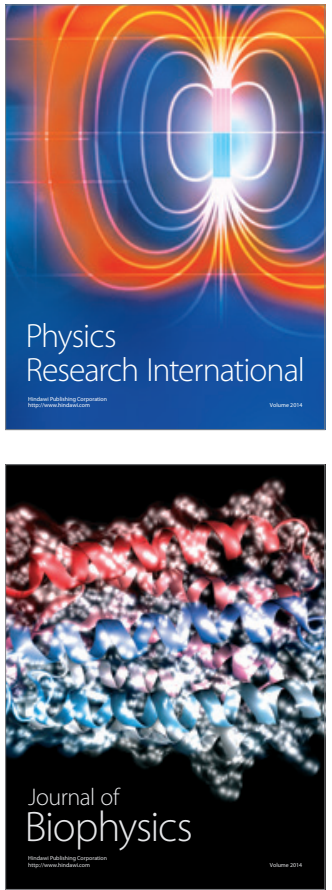
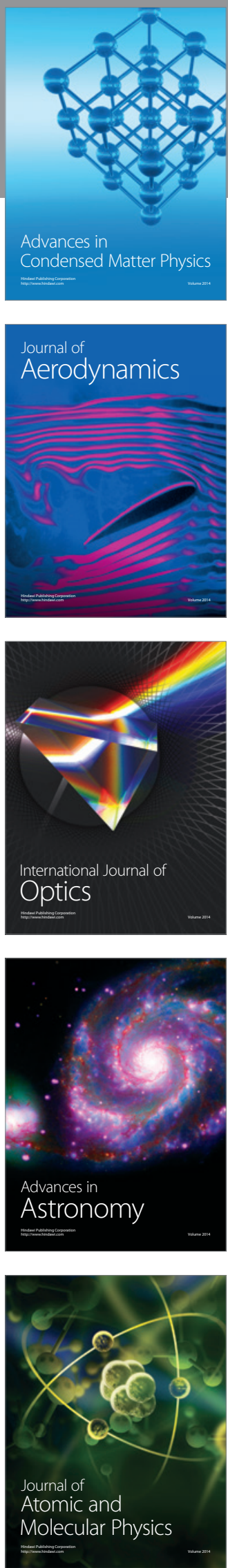